\title{
Modeling a Driver's Directional and Longitudinal Speed Control Based on Racing Track Features
}

\author{
Jianfeng Wang $\mathbb{D},{ }^{1,2}$ Weihua $\mathrm{Li}\left(\mathbb{D},{ }^{1} \mathrm{Jun} \mathrm{Li}^{2}{ }^{2}\right.$ Yiqun Liu $\mathbb{D}^{1},{ }^{1}$ Baoyu Song, ${ }^{2}$ and Haibo Gao ${ }^{2}$ \\ ${ }^{1}$ School of Automotive Engineering, Harbin Institute of Technology, Weihai, Shandong, China \\ ${ }^{2}$ State Key Laboratory of Robotics and System, Harbin Institute of Technology, Harbin, China \\ Correspondence should be addressed to Weihua Li; liweihua.08301@163.com
}

Received 19 October 2017; Accepted 1 April 2018; Published 8 May 2018

Academic Editor: Onome E. Scott-Emuakpor

Copyright (c) 2018 Jianfeng Wang et al. This is an open access article distributed under the Creative Commons Attribution License, which permits unrestricted use, distribution, and reproduction in any medium, provided the original work is properly cited.

\begin{abstract}
This study firstly analyses the driver's manipulation behaviour and relates the different components of the driver model. Then, a model controlling the driver directions is built according to the prediction-follower theory with the aim of improving the point search algorithm. A model of the driving system of an electric vehicle is used to establish the longitudinal speed control model of the driver by using a feedforward-PID feedback control strategy. Our approach is to release the coupling between direction and speed control and build an integrated model that includes the direction and speed for an arbitrary path. Finally, the characteristics of an actual racing track are considered to establish the fastest driver control model. We simulated the typical operating conditions of our driver operation model. The simulation confirmed the effectiveness of the improved predictive point search algorithm and the integrated driver model to control the direction and speed for an arbitrary path.
\end{abstract}

\section{Introduction}

The study of driver behaviour is an important part of vehicle dynamics and control research. Additionally, it is also an important basis for the closed-loop evaluation of vehicle control stability and the development of a vehicle-assisted driving system [1]. When manipulating the vehicle, the driver's behaviour is a process consisting of simultaneously adjusting the direction and speed of the vehicle. This enables the vehicle to proceed safely in accordance with the driver's intention. Driving behaviour mainly includes two aspects, namely, vehicle direction control and vehicle speed control. In this regard, the prediction-follower theory is a highly effective method to establish the driver model. Currently, driver models that are widely used in passenger cars for the driver direction control and driver speed control are established based on neural network theory or simple PID control. However, this approach is only suitable for paths with a small amount of curvature. A racing track, which is characterised by a high adhesion coefficient and large curvature, is quite different from an ordinary road. Therefore, the driver model for passenger cars is not fully applicable to racing cars. It is thus highly important to establish a driver control model based on the characteristics of the racing driver for the development of a car control system.

Driver manipulation of the vehicle can be divided into three parts, namely, information perception, prediction decision, and control correction, as shown in Figure 1. The information perception module describes the driver's perception and understanding of the information such as the driving environment of the road outside and the state of vehicle motion. It provides the driver with effective road environment data and information about the state of the vehicle, as well as the database of the prediction decision module and the control calibration module. At present, the perception of the environment surrounding the vehicle mainly depends on the combined use of distance-measuring and visual sensors. The information provided by the distancemeasuring sensor is accurate and real-time, whereas the visual sensor collects richer information, because its algorithm is more mature. A combination of the two would be highly effective. Multiple sensors are often used to measure the same object under complex conditions, in which case a random class method, such as the weighted mean method, 


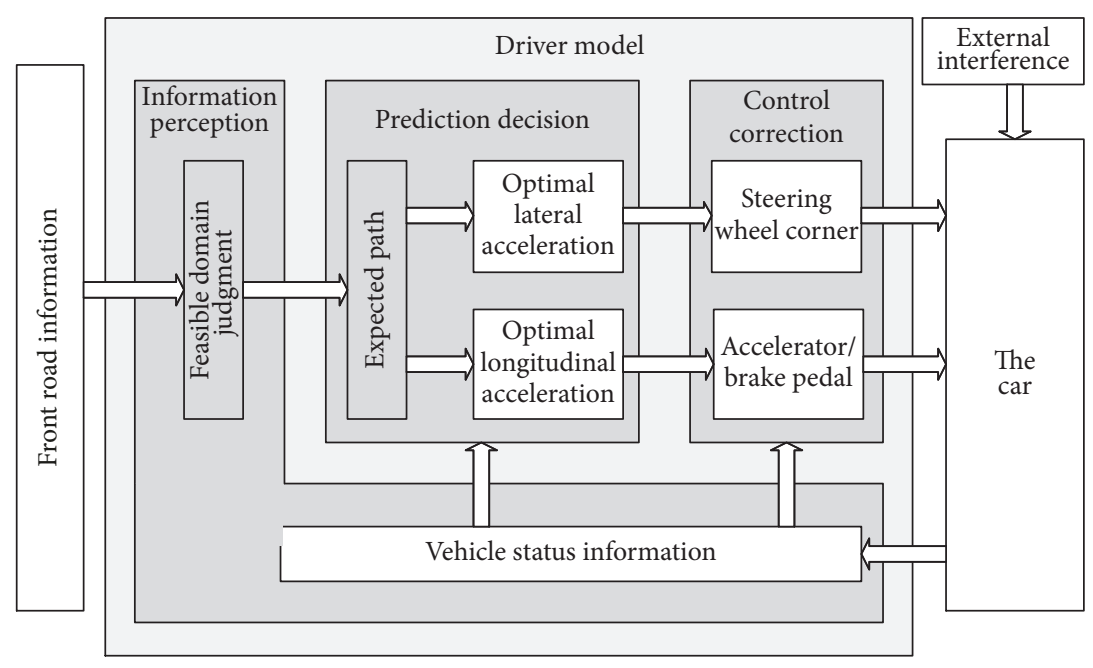

FIGURE 1: Basic structure of the driver model.

multi-Bayesian estimation method, and intelligent control method (e.g., fuzzy logic reasoning and a neural network), is used for data fusion [2-4]. Vehicle information, such as its location, yaw angle, centre of mass angle, longitudinal velocity, lateral velocity, longitudinal acceleration, lateral acceleration, and wheel speed, is very important for the design of a vehicle chassis control system. The various vehicle information estimates are obtained by integrating the GPS, INS, and wheel speed information and necessitate the use of a Kalman filter [5].

The decision module simulates the process by which the driver chooses the best course of action from among a number of viable alternatives [6]. For racing, the decision-making process can be simplified as the shortest single-objective decision-making problem. Decision-making methods that are commonly used are fuzzy reasoning, neural networks, machine learning, and so on. All of these algorithms are adaptive with the ability to learn.

The control calibration module simulates the process by which the driver manoeuvres the vehicle toward the desired position through the steering wheel, the accelerator pedal, and the brake pedal. The driver's manipulation control action is the reason for the change in the movement state of the vehicle.

Domestic and foreign scholars have studied the driver model in different ways. Sentouh et al. attempted to construct a cybernetic model of a driver steering road vehicle in order to make the sensorimotor dynamics explicit [7]. Apart from the visual and kinesthetic perception, compensatory and anticipatory processes were also taken into account in this proposed model, which extended previous work. The unknown parameters of the model were identified using the grey box identification concept to simulate car trajectories. The driver-vehicle system was then analysed in order to improve parameter sensitivity. Yang and Peng presented an error-inducing car-following driver model, which was developed for the evaluation and design of active safety systems [8]. They obtained car-following data from a largescale naturalistic driving database to develop this model. Three error-inducing behaviours were introduced in this paper, by which rear-end collisions with the lead vehicle could occur. Gray et al. enforced safety constraints with minimal control intervention by using a robust Model Predictive Controller (MPC) [9]. The robust MPC computes the smallest corrective steering action required to ensure the safety of the driver for all predicted trajectories. The effect of the proposed framework was shown in simulations of a driver approaching multiple obstacles. By previewing the path ahead of the vehicle, Chatzikomis and Spentzas presented a combined longitudinal-lateral controller, which regulated the steering angle and throttle/brake levels [10]. The heading and position deviation between the vehicle and the road were used as input in the lateral steering controller. The curvature of the path ahead of the vehicle was used in the longitudinal speed controller in order to determine the appropriate velocity. Menhour et al. proposed a mathematical driver model combined with 2-DOF PID multiple controllers [11]. Linear time invariant models were used to construct this model synthesised by the Ziegler-Nichols oscillation method. $\mathrm{Li}$ et al. presented a new comprehensive driver model for critical manoeuvring conditions with more accurate dynamic control performance [12]. A new path-planning scheme was designed to maintain vehicle stability. Simulation was used to validate the control performance compared with the optimal preview driver model proposed before. Mikami et al. developed a personalised driver assisting system, which made use of a driver behaviour model [13]. They produced the Probability-weighted ARX (PrARX) model, as a driving behaviour model, to describe the driver's vehiclefollowing skill. This model was based on a simple gradient descent algorithm using actual driving data from a driving simulator.

This paper firstly improves the prediction point search algorithm on the basis of the propulsion optimisation 


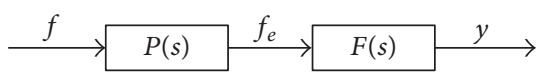

FIGURe 2: Prediction-follower system.

artificial neural network driver direction control model, making it suitable to follow a path with a large amount of curvature. Subsequently, a driver direction control model is established for racing drivers. We overcame the shortcomings of PID control (slow response and large overshoot of driver speed control model) by using a feedforward-PID feedback compound control strategy to establish the driver speed control model. Furthermore, by releasing the coupling between the longitudinal and lateral dynamic characteristics, we built the integrated control driver model of direction and speed for racing along an arbitrary path. Finally, in terms of the racing characteristics, the control driver model capable of supporting the fastest speed is established.

\section{Establishment of Driver Direction Control Model}

The driver-vehicle closed-loop system could be understood as a system for following control based on the input information of the previous path. This system could be modeled as a tandem system with a previewer and a follower, known as a prediction-follower system [14], as shown in Figure 2. A propulsion optimisation artificial neural network driver model was proposed by analysing the prediction-follow system process and by combining artificial neural network topology [15].

In practice, the driver could observe the front path information in advance; thus the multipoint prediction method was used in the simulation. Because the driver could not take action immediately when receiving the pavement information, hysteresis and a first-order inertia link was used in the simulation. In order to simplify the prediction artificial neural network driver model to reduce the calculation time of the model, this study considered a single-layer neural network instead of the original neural network topology [16]. According to [16], the prediction was further simplified as shown in Figure 3.

The dynamics of the vehicle was studied by simultaneously simplifying the model. The vehicle could be simplified as a 2 -DOF (degree-of-freedom) system $[1,17]$. The model parameters were determined by using an analytical method for error analysis to compute the parameters of the driver's direction control model [17], as shown in Figure 4. This allowed the driver direction control model to obtain the weight value and other parameters by the vehicle parameters, driver lag time constant, and prediction time. This greatly simplified the parameter acquisition process of the model, which increased the applicability of the model.

The application of the simplified preview optimisation artificial neural network driver direction control model requires the vehicle model established in Carsim, a vehicle dynamics simulation software of MSC company in USA, to be equivalent to a 2-DOF vehicle model. The methods were as follows. In this study, we selected the trapezoidal signal as the input signal of the Carsim vehicle model steering wheel angle to excite the frequency characteristics of the Carsim vehicle model, as shown in Figure 5(a). The signal was inputted to the 2-DOF vehicle model and the Carsim vehicle model. The lateral accelerations of the 2-DOF vehicle model and the Carsim vehicle model, $\widetilde{a}_{y}$ and $a_{y}$ were extracted. The difference between them, $a_{y}-\widetilde{a}_{y}$, was set as the objective function and the parameters of the model were obtained by the optimisation method shown in

$$
J=\int_{0}^{t}\left(a_{y}-\tilde{a}_{y}\right)^{2} d t .
$$

The identification results of the Carsim vehicle model at different vehicle speeds are shown in Figures 5(b) and 5 (c). The results in the two figures indicate that the output of the 2-DOF vehicle model could be consistent with the output of the Carsim vehicle model over a large vehicle speed range.

Previously, researchers $[18,19]$ used a discrete number table to describe any path and proposed a prediction point search algorithm for the small curvature path. This method transformed the trajectory following the time domain into that following the spatial domain. Figure 6 showed the eightshaped surrounding track of Fortinet Server Authentication Extension (FSAE), which was simplified for verification of the prediction point search algorithm. The HRT-15E racing car model built in Carsim was used and the speed was $40 \mathrm{~km} / \mathrm{h}$. The simulation result is shown in Figure 8. The simulation represented by the red solid line showed that the vehicle travel path terminated at a larger curvature and that the next prediction point could not be correctly identified within the field of view after the current prediction point. The results proved that although this prediction point search method suited the small curvature path well, it would not meet the requirements of a racing track with high curvature.

To solve the problem presented by tracking a path with a large amount of curvature, we improved the prediction search algorithm, as shown in Figure 7. The basic flow of the improved prediction search algorithm is as follows:

(1) finding the nearest point in the current position of the vehicle as the starting point of the next search in the table showing the centre line of the road ahead;

(2) finding two adjacent points in the table, setting the preview point exactly between the two points, then using a linear interpolation method to obtain the prediction point, and converting it into the vehicle coordinate system.

The same method was used to search the vehicle location [19], as shown in (2), although the method to search for the prediction point was different as shown in Figure 7.

$$
\left[x(m)-v_{x} T\right]\left[x(m+1)-v_{x} T\right] \leq 0 .
$$

The distance covered by the vehicle should be equal to the vehicle travel distance during the prediction time $T_{p}$, as follows:

$$
S_{d}=v_{x} \cdot T_{p}
$$




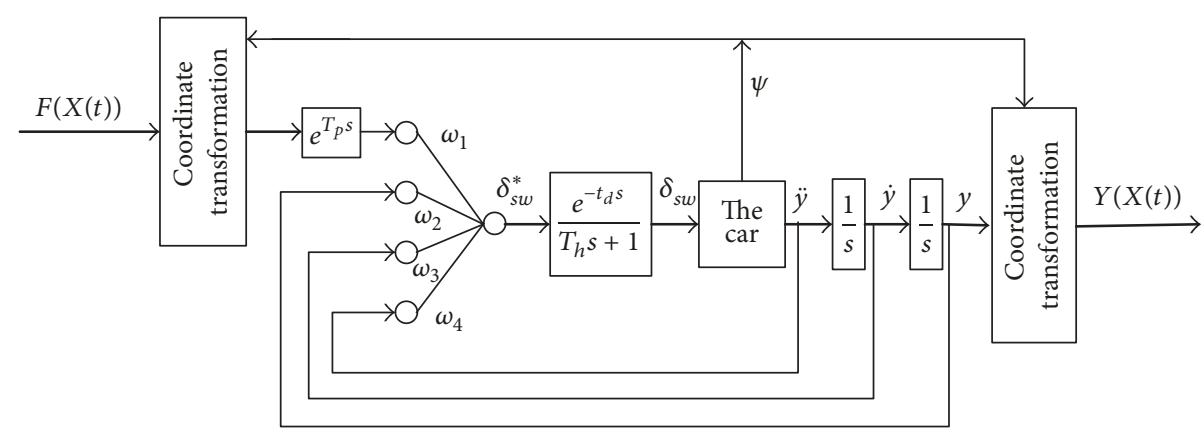

FIGURE 3: Simplified model [16].

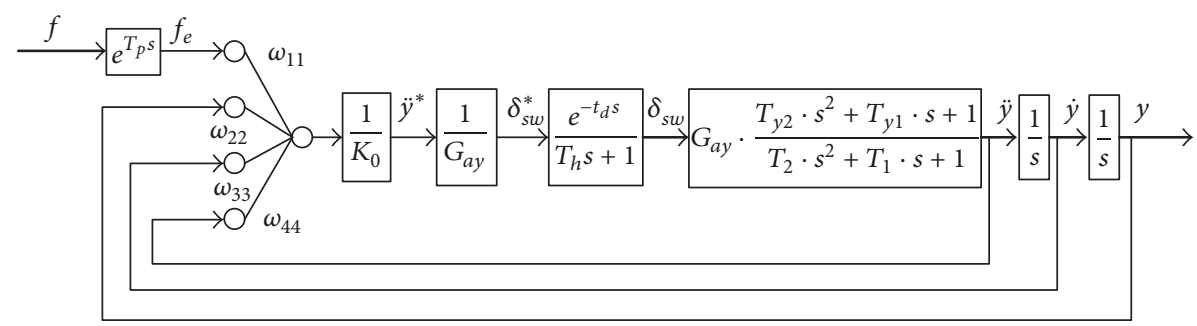

FIGURE 4: Error analysis method.

Thus, the closest point in the prediction trajectory after the current point could be determined by

$$
\begin{aligned}
& {\left[\sum_{j=i_{0}}^{m} \sqrt{(x(j+1)-x(j))^{2}+(y(j+1)-y(j))^{2}}\right.} \\
& \left.-v_{x} T\right] \\
& \cdot\left[\sum_{j=i_{0}}^{m+1} \sqrt{(x(j+1)-x(j))^{2}+(y(j+1)-y(j))^{2}}\right. \\
& \left.-v_{x} T\right] \leq 0 .
\end{aligned}
$$

Further, $\Delta f_{p}$ could still be obtained by the linear interpolation method of the coordinates of point $m$ and point $m+1$ in the vehicle coordinate system.

$$
\Delta f_{p}=y(m)+\frac{y(m+1)-y(m)}{x(m+1)-x(m)}\left[v_{x} T-x(m)\right] .
$$

Again simulating under the same condition as before produces the result shown in Figure 8. Clearly, the improved prediction point search algorithm could track the large curvature path well.

\section{Driver Direction Control Model}

The specific structure of the HRT-15E racing power transmission system we studied as shown in Figure 9. The power drive system is mainly composed of the motor and transmission system, with the torque provided by the motor responsible for powering the vehicle through the transmission system to the drive wheel. The HRT-15E electric racing motor was a high-pressure water-cooled three-phase permanent magnet synchronous motor, named EMRAX 228, of the Enstroj company, Slovenia, with a motor controller based on SVPWM (Space Vector Pulse Width Modulation) technology. The motor characteristic curve is shown in Figure 10.

Because of the complexity of the model of the motor dynamics [20], in this research we ignored the dynamic characteristics of the motor and were only concerned about the input and output characteristics of the motor and its controller.

The vehicle chassis control system, including vehicle direction control and vehicle speed control, is controlled by the driver, who controls the vehicle via the steering wheel, accelerator pedal, and brake pedal. The controlled system, which was a strongly nonlinear system with timevarying parameters, made it difficult to build the model precisely. In this study, a linear approximation method was used to linearise the strong nonlinearity of the vehicle near its equilibrium point $[21,22]$. The effective input $\sigma$ of the accelerator pedal and brake pedal was taken as the input of the power drive unit. The motor output torque $T_{m}$ was taken as the output of the power drive unit. The relationship between 


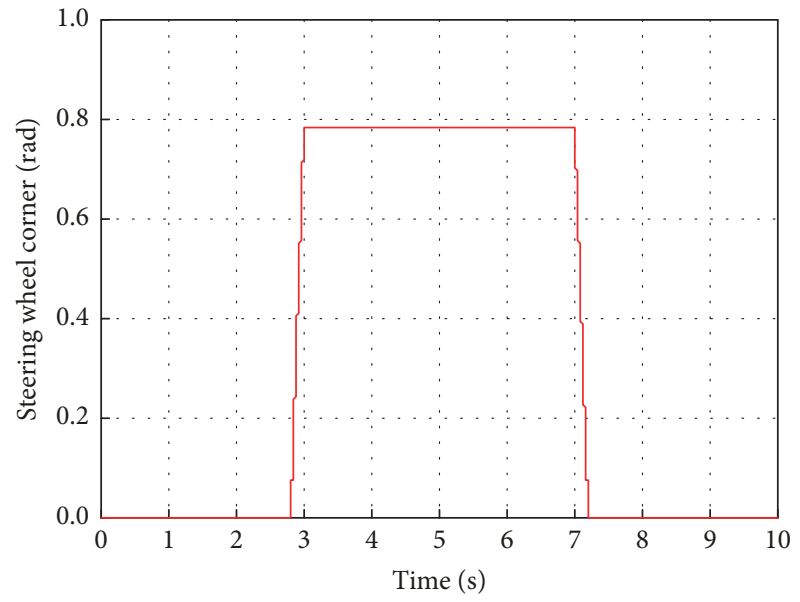

a)

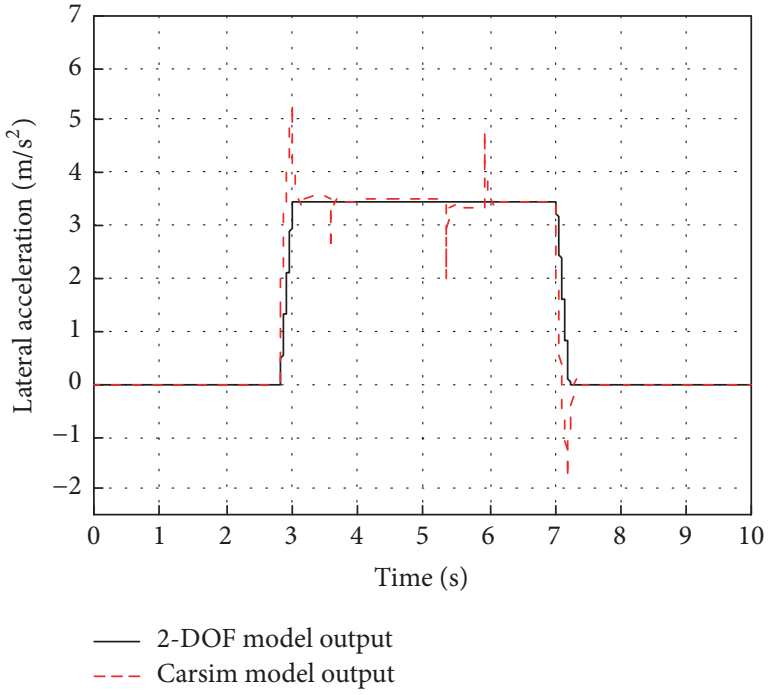

(b)

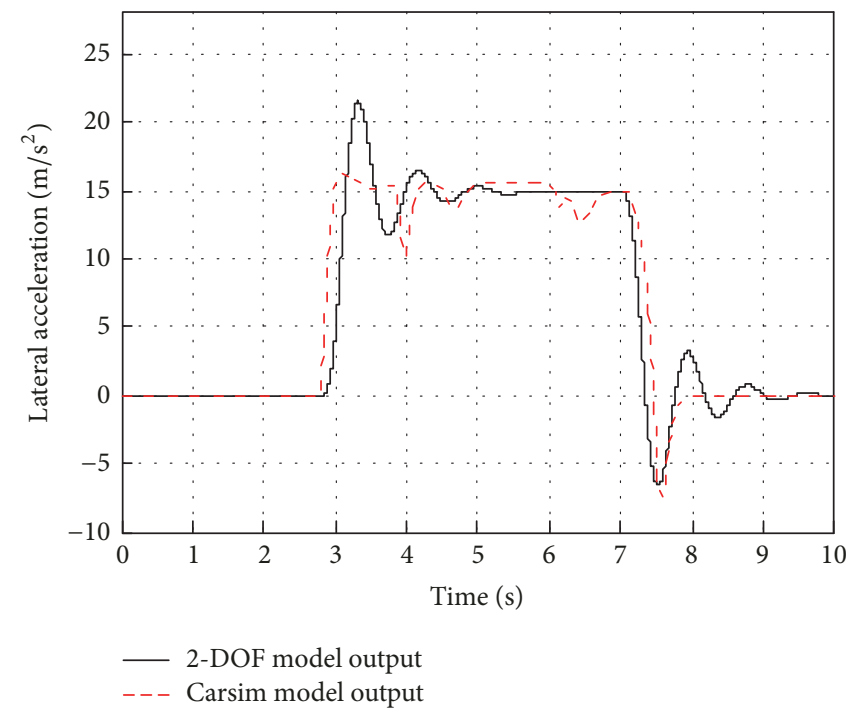

(c)

Figure 5: Identification results of the Carsim vehicle model. (a) Input signal. (b) $v=20 \mathrm{~km} / \mathrm{h}$. (c) $v=90 \mathrm{~km} / \mathrm{h}$.

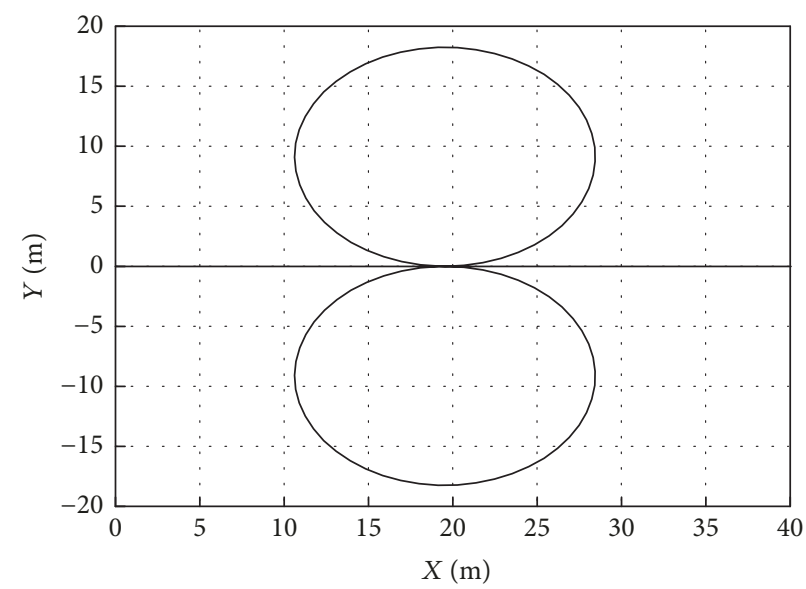

FIGURE 6: Figure-eight-shaped continuously looping track. 


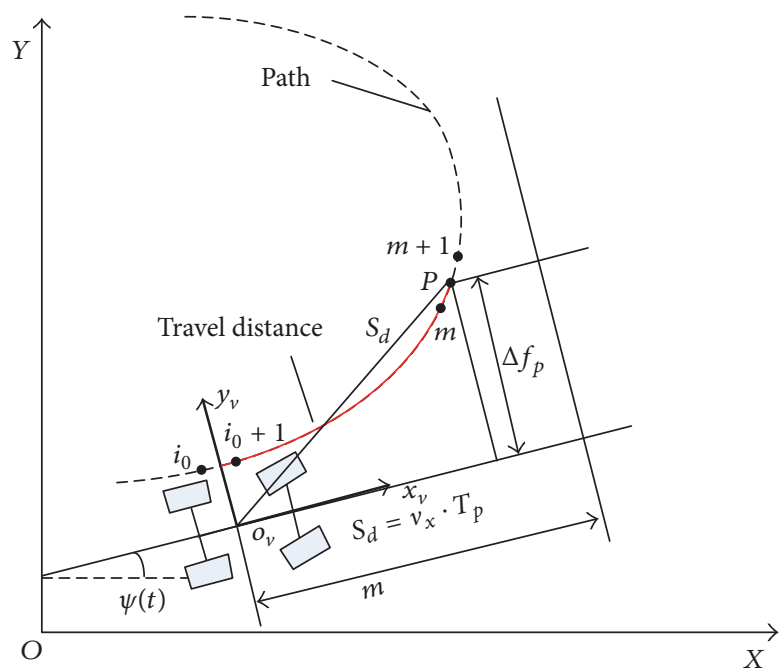

FIGURE 7: Method of tracking a path with a large amount of curvature.

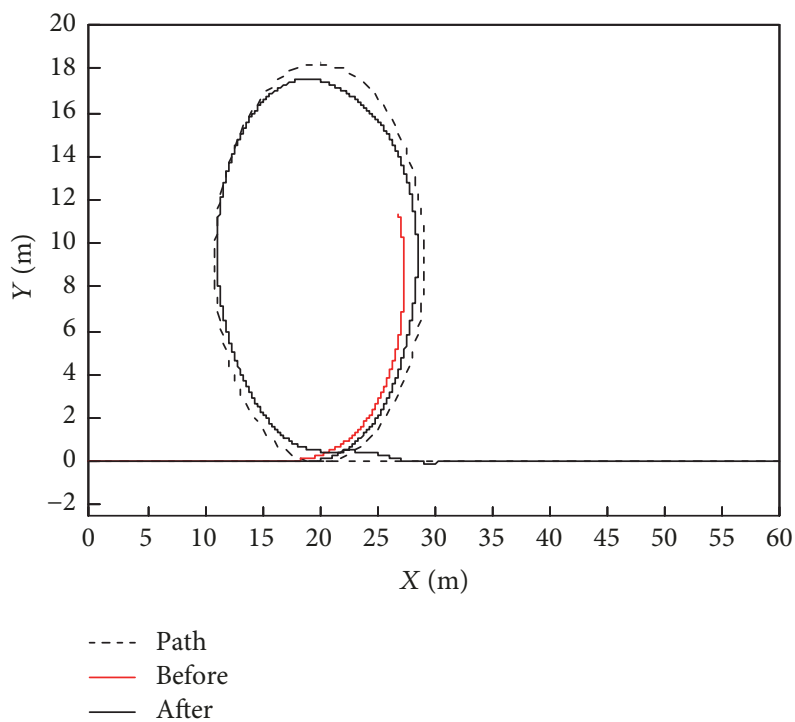

FIGURE 8: Simulation result of improved prediction point search algorithm.

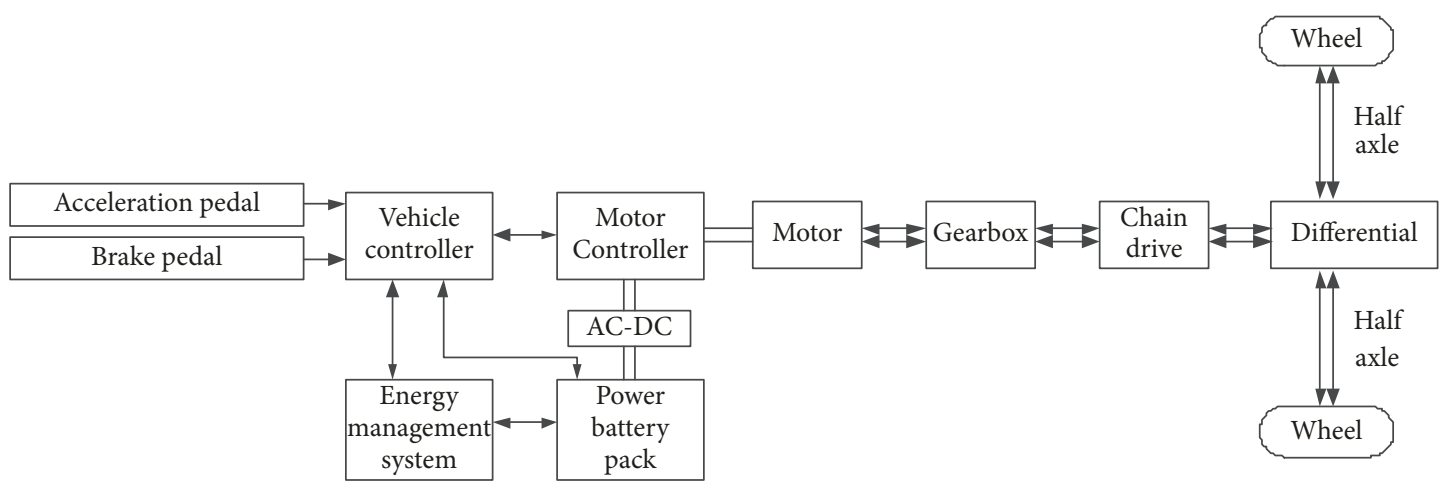

$\longleftrightarrow$ Signal

$\rightleftarrows$ Mechanical connection

Electrical connections

FIGURE 9: HRT-15E racing power transmission system. 


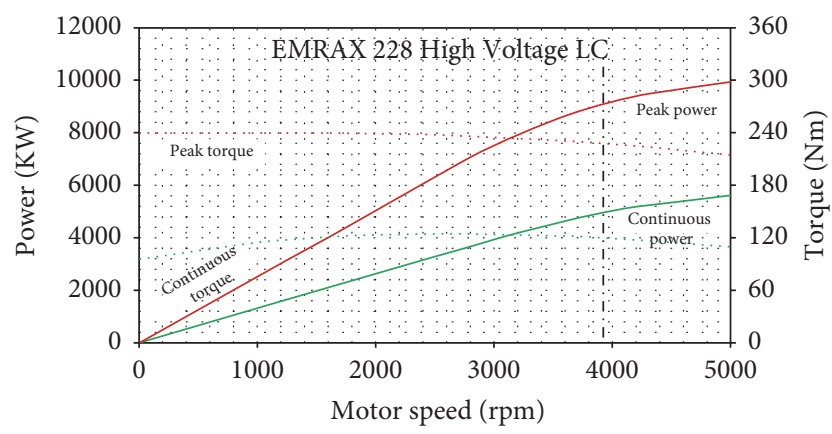

FIGURE 10: Motor characteristic curve.

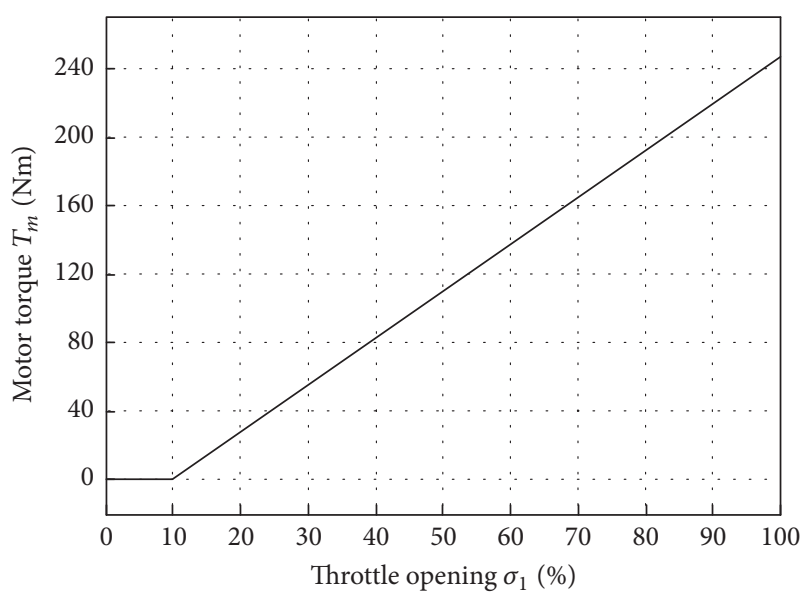

FIgURE 11: Throttle opening and motor output torque curve.

the throttle opening and the motor output torque, based on the design of the HRT-15E electric racing power drive unit, is shown in Figure 11 and is expressed by

$$
T_{m}= \begin{cases}0 & 0 \leq \sigma_{1} \leq 0.1, \\ 273\left(\sigma_{1}-0.1\right) & 0.1 \leq \sigma_{1} \leq 1 .\end{cases}
$$

The throttle was prevented from opening during an empty stroke by tuning the throttle opening $\sigma_{1}$ according to the effective input $\sigma$ of the accelerator pedal. The relationship between them was $\sigma_{1}=0.1+0.9 \sigma$. Hence, the relationship between the effective input of the accelerator pedal and the output torque $T_{m}$ of the motor was $T_{m}=245 \sigma$. It was difficult to test the relationship between the output torque of the motor and the effective input of the accelerator pedal, because of the lack of test equipment; thus, dynamic items were ignored.

This study mainly investigated the driving behaviour of the driver. Therefore, the transmission system was regarded as rigid, regardless of the torque and energy loss of the transmission process. The HRT-15E electric racing transmission system mainly included a transmission chain, antiskid differential, drive shaft, and wheel. Among them, the transmission chain played the role of transmission and main reducer in traditional vehicles with an internal combustion engine. According to the number of teeth of the small and large

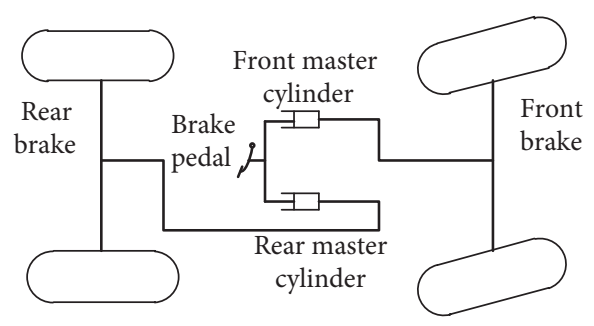

FIgURE 12: Dual-loop braking system in HRT-15E.

sprockets, the transmission ratio should be 3.077. The antislip differential only had a torque distribution function. This paper focused on the torque input and output characteristics of the power drive system. The motor output torque $T_{m}$ and the driving torque $T_{e}$ acting on the drive shaft satisfied the relationship in (7), which is the transmission system model:

$$
T_{e}=i \cdot T_{m}
$$

To meet FSAE rules, a dual-loop braking system was used in HRT-15E, as shown in Figure 12. This system facilitated adjustment of the front and rear wheel braking force ratio. According to the braking system design scheme, the ratio of the front and rear wheel braking force was $4: 6$.

In the simplified prediction optimisation model of artificial neural network driver direction control, movement of the vehicle in the prediction time was assumed to be uniform acceleration movement [15], even though this is not the case in reality. In previous work [23], it was assumed that during the period of prediction time, the movement could be regarded as uniform acceleration, provided the speed did not change dramatically. On this basis, a PID control strategy could be used to achieve longitudinal velocity control [22, 24]. Considering the driver's nerve response lag time $t_{d}$ and inertia lag time $t_{h}$, a lag $e^{-t_{d} s} /\left(t_{h} s+1\right)$ was connected in series with the controller. The control strategy is depicted in Figure 13.

A longitudinal speed control simulation system for the HRT-15E electric racer was built based on the SimulinkCarsim simulation platform, which was the same as the system removing $G_{f 1}$ and $G_{f 2}$ in Figure 14. The expected speed tracking results are shown in Figure 15(a), which indicates that the tracking response time of the simple PID was slightly longer and did not meet the fast response characteristics of a racing driver. Thus, we built a longitudinal velocity control strategy combined with feedforward-PID feedback, as shown in Figure 14. The feedback, $G_{f 1}$ and $G_{f 2}$, could be obtained by analysis of the control system.

The simulation results are presented in Figure 15(b). The response time and overshoot of the improved model were both less than that of model based only on PID feedback under the same step signal. Therefore, a longitudinal speed control strategy combined with feedforward-PID feedback could be effectively used in the development of an electric racing chassis control system. 


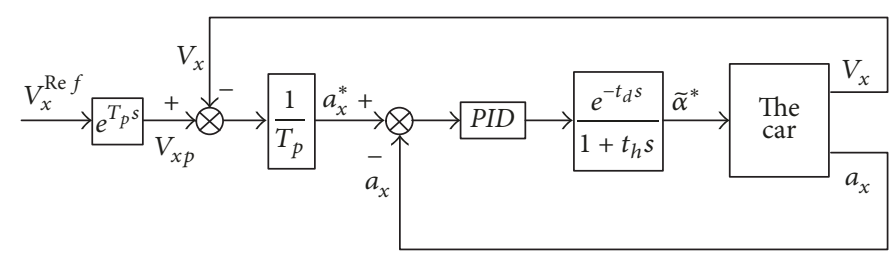

FIGURE 13: Longitudinal speed control strategy [18].

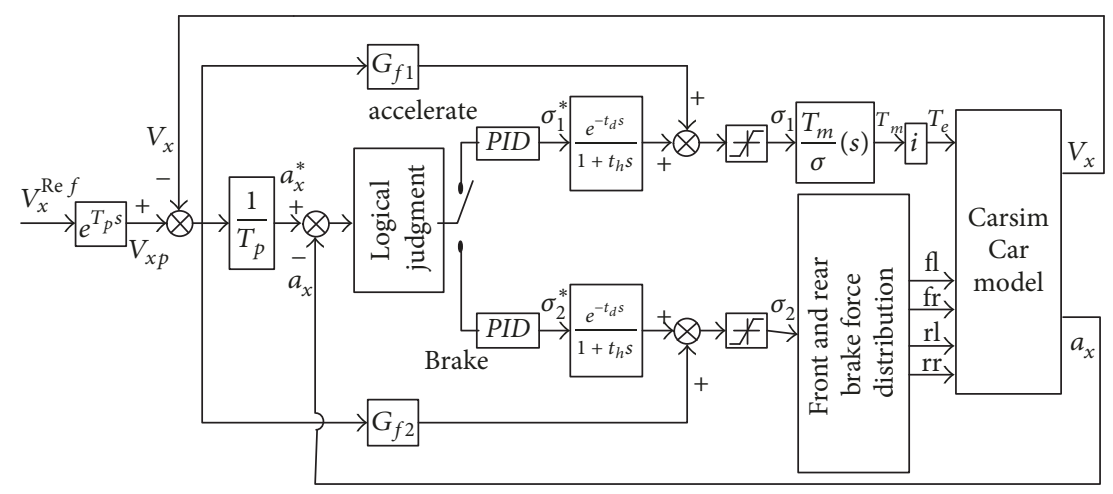

FIGURE 14: Longitudinal speed control strategy combined with feedforward-PID feedback.

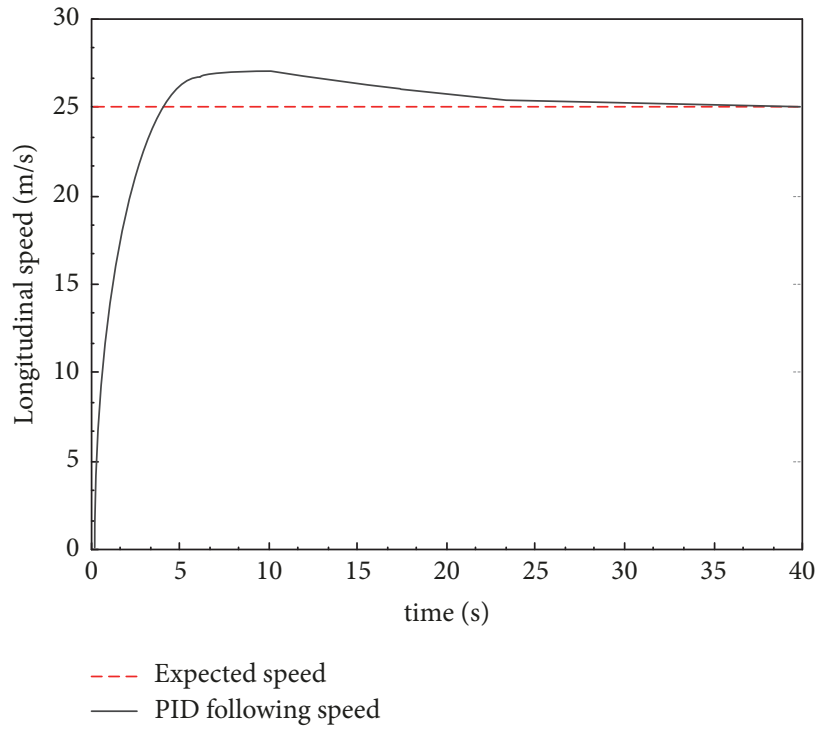

(a)

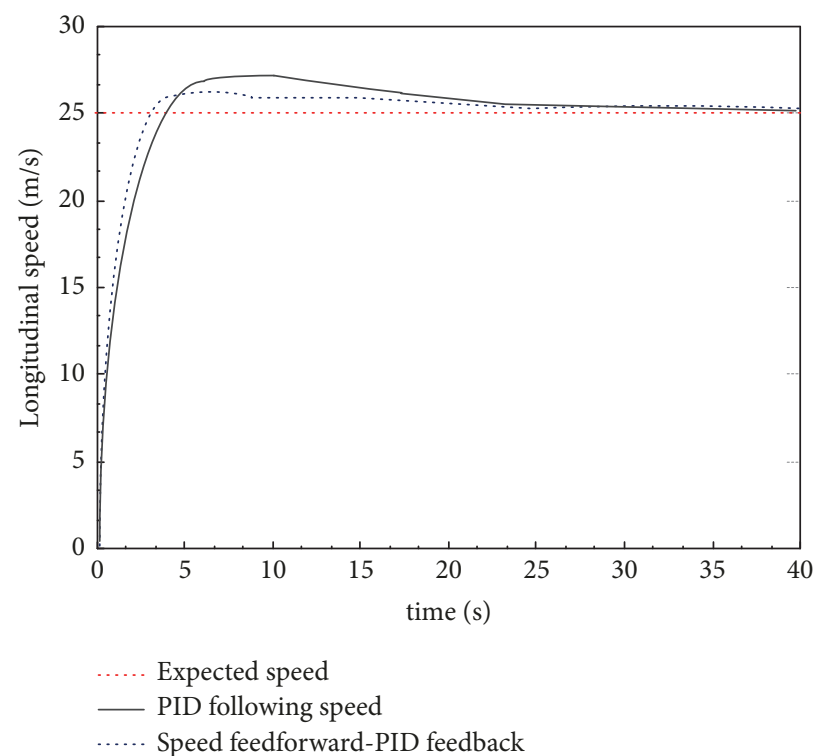

(b)

FIGURE 15: Simulation results.

\section{Model Decoupling of Driver Direction and Longitudinal Speed Integrated Control}

The driver's actual driving behaviour is more diverse as could be simulated by single speed or direction control. Thus, we should establish a driver model of direction and longitudinal speed integrated control. In addition, changes in the vehicle speed would often lead to changes in the vehicle model parameters, and this requires the corresponding control parameters in the driver control model to be changed.

Based on the above content, we established the driver direction and longitudinal speed integrated control model, as shown in Figure 16. 


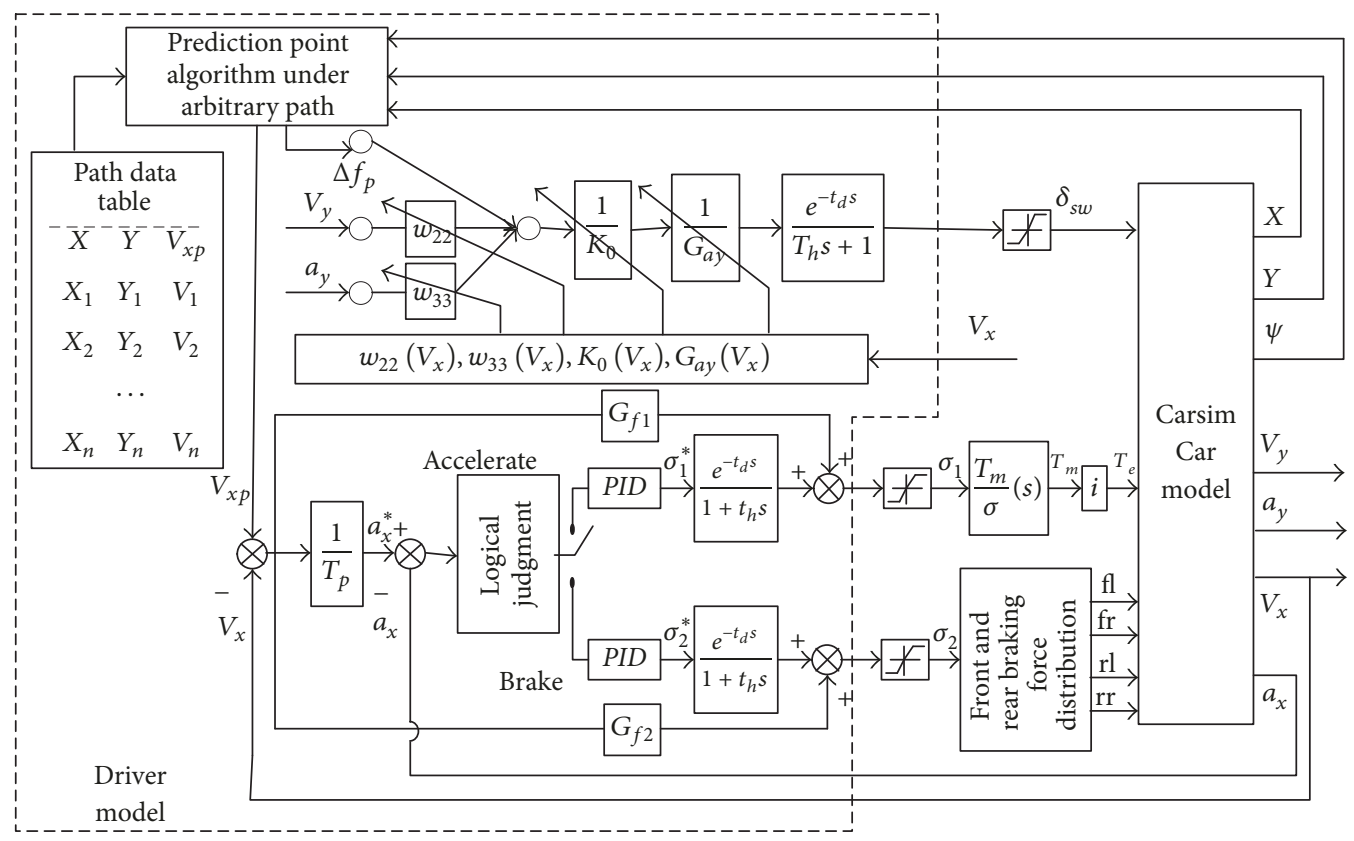

FIGURE 16: Direction and longitudinal speed integrated control model.

It is known that the coupling between the parameters representing the lateral and longitudinal characteristics of the vehicle affects the parameters of the direction control model or the longitudinal speed control model $[25,26]$. Owing to the difficulty in controlling the coupling system, it was desirable to decouple the coupling system into multiple independent single-input and single-output subsystems by realising that a certain output was only controlled by a certain input [27]. Researchers have previously pointed out [18] that in a shorter prediction time interval, because of smaller changes in the vehicle status, the influence of the steering wheel on the longitudinal characteristics was small and could be ignored.

The longitudinal velocity of the vehicle had a large effect on the lateral characteristics of the vehicle. The lateral gain $G_{a y}$ could be fitted to the function of longitudinal steady-state gain $V_{x}$ on the steering wheel angle. The above-mentioned parameter identification method was used to conduct an equivalent 2 -DOF vehicle model parameter identification. These identification results were transformed into the lateral acceleration $G_{a y}$ on the steering wheel steady-state gain, the functional relationship between the vehicle dynamic parameters, $T_{q 1}$ and $T_{q 2}$, and longitudinal velocity $V_{x}$. The fitting results are shown in Figure 17. As for $T_{q 1}$, the fitting result was negative at the time $V_{x}=0 \mathrm{~km} / \mathrm{h}$, which was obviously unreasonable. Therefore, a compensation gain was added to correct the result, as shown in Figure 17(d).

Finally, our aim was to use the idea of ensuring local optimal optimisation to achieve the global optimum as far as possible to enable the vehicle on a local path to attain a high speed to suit the characteristics of racing. This process was repeated to achieve the shortest travel time of the vehicle for the overall path.

\section{Simulation Analysis in Typical Operating Conditions}

For this research, we chose the durable track located in Xiangyang, China, which is one of the most complex racing tracks in China, as a typical working condition. The main simulation parameters were set as follows: the friction coefficient between the hot melt and the track is specified as $\mu=1.5$, and the vehicle speed when running resistance was balanced with the maximum driving force of the vehicle: $V_{b}=75 \mathrm{~km} / \mathrm{h}$.

The simulation results are shown in Figure 18. The electric racing car was rapidly accelerated from stationary to the highest speed and then decelerated (accelerated) as the curvature increased (decreased) until the mission was completed. The result in Figure 18(a) indicates that the model could track the large curvature path well, and the tracking deviation was very small.

Figure 18(b) shows that the lateral acceleration during the whole simulation process was less than $15 \mathrm{~m} / \mathrm{s}^{2}$ and Figure 18(c) shows that the longitudinal velocity varies with the curvature of the curve. In Figure 18(d), it can be seen that, at the larger curvature, the required steering wheel angle calculated theoretically exceeded the maximum value of the HRT-15E steering system; as a result, the saturation value was selected as the actual steering wheel input.

The simulation results of the durability track in Xiangyang confirmed that the final model of the racing driver could successfully complete the tracking task of a track with a large amount of curvature. The model could be further used for the development of an electric racing chassis control system. 


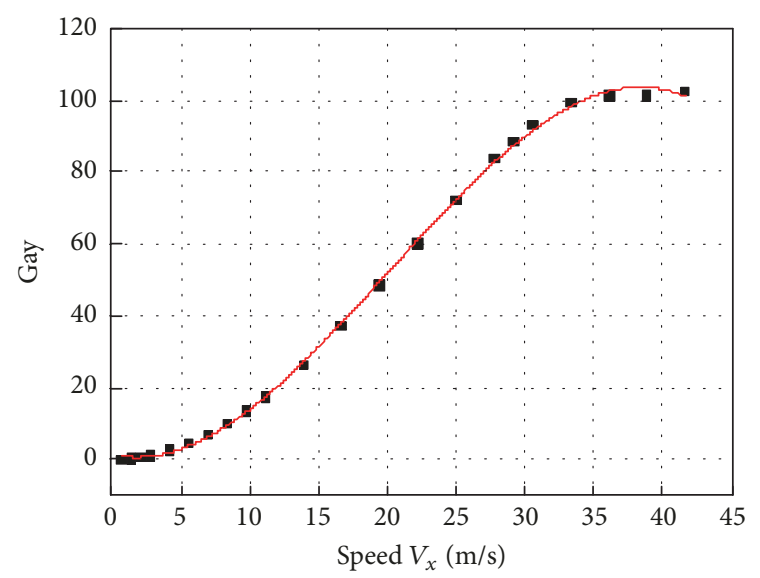

- Gay

— Gay fitting

(a)

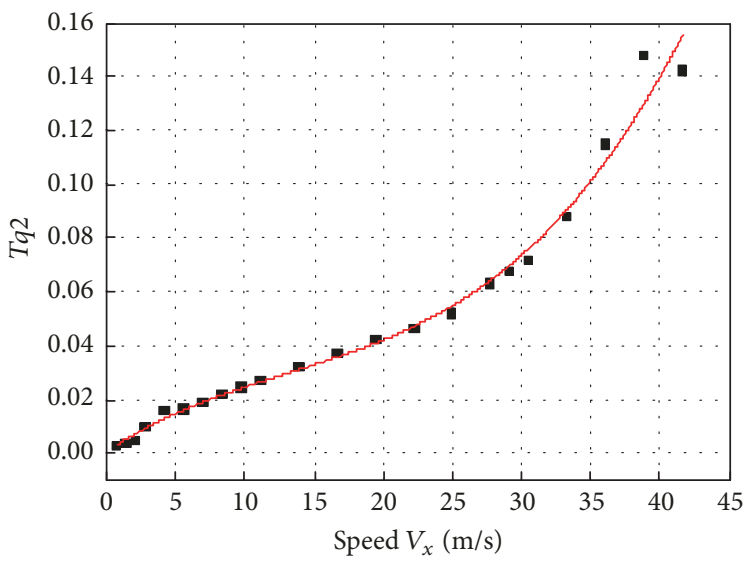

- $T q^{2}$

- Tq2 fitting

(c)

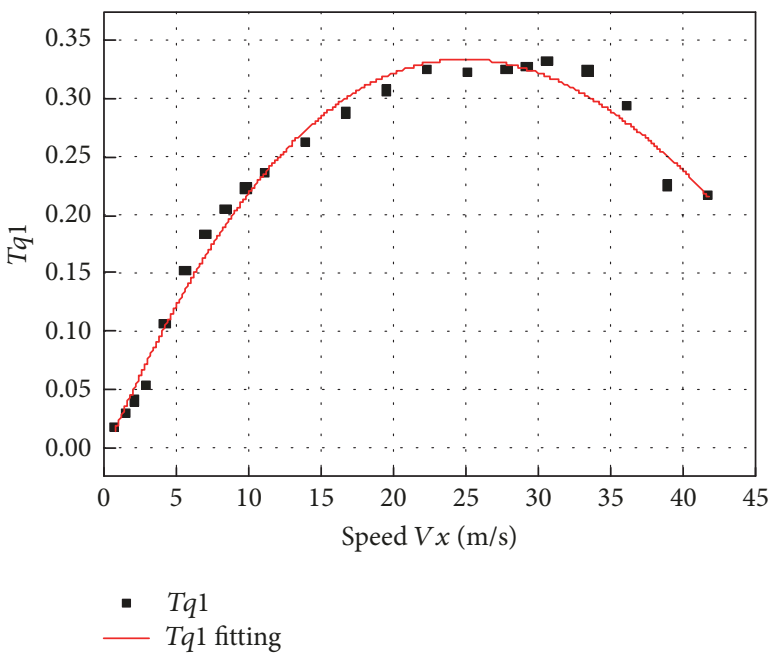

(b)

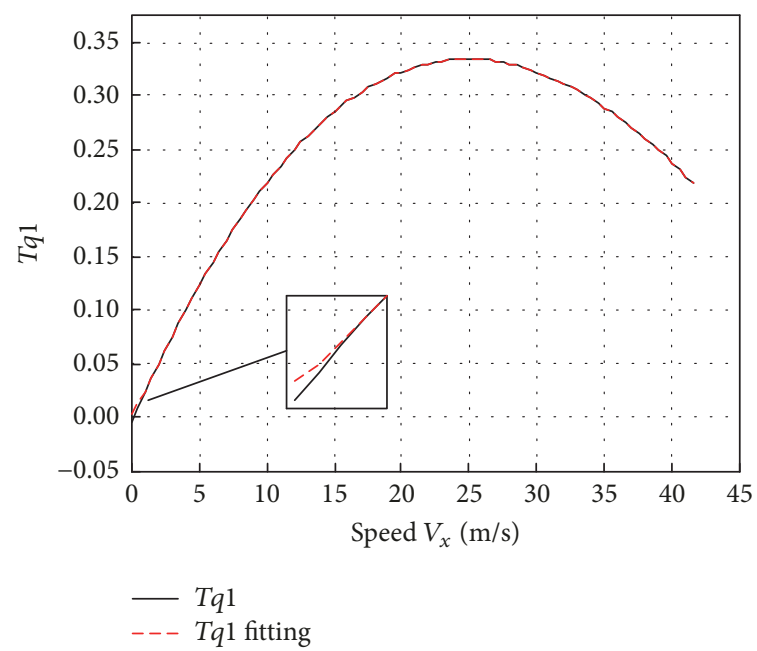

(d)

FIGURE 17: Fitting results. (a) $G_{a y}$, (b) $T_{q 1}$, (c) $T_{q 2}$, (d) $T_{q 1}$ correcting.

\section{Conclusion}

Based on the preview-follower theory, the improved preview point search algorithm is used to establish a driver direction control model suitable for racing cars. The tracking simulation of an figure-eight-shaped continuously looping track was used to prove that the driver direction control model can successfully track a path with a large amount of curvature.

On the basis of the power drive system model, the driver longitudinal speed control model was established by implementing feedforward-PID feedback. Simulation under the same step signal proved that the longitudinal velocity control model has a smaller response time and overshoot, which is more accurate than the model without improvement.

The driver model presented in this paper was constructed by combining the two driver models and a decoupling system, after which this combination was integrated with the fastest speed control driver model, which achieves the shortest global travel time. Simulation of the durable track in Xiangyang, China, proved that the final model is excellent for completing a track with considerable curvature. Thus, the model can be effectively used in the development of an electric racing chassis control system.

\section{Conflicts of Interest}

The authors declare that there are no conflicts of interest regarding the publication of this paper.

\section{Acknowledgments}

This study was supported in part by the Foundation of Chinese State Key Laboratory of Robotics and Systems (Grant no. SKLRS201602B). 


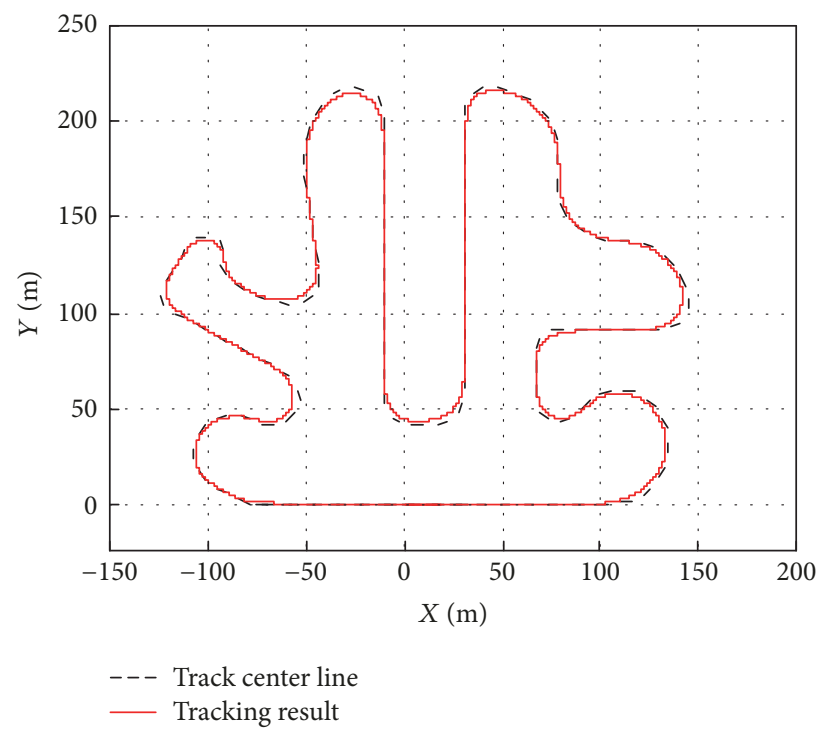

(a)

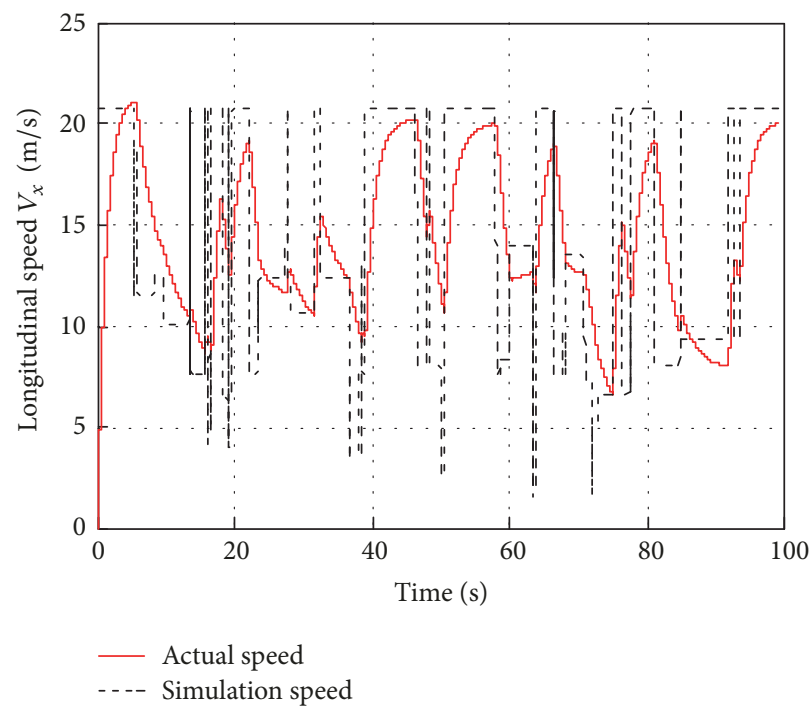

(c)

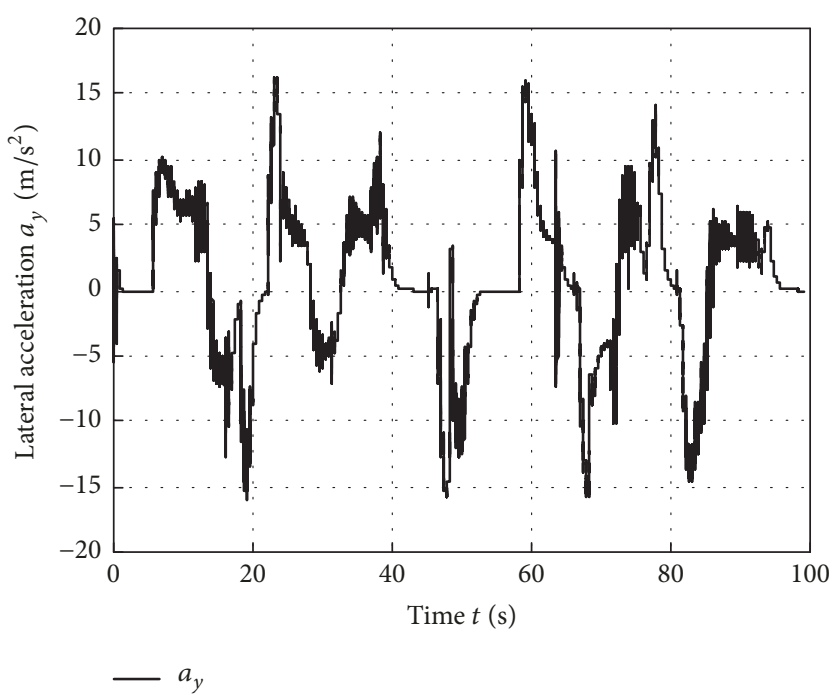

(b)

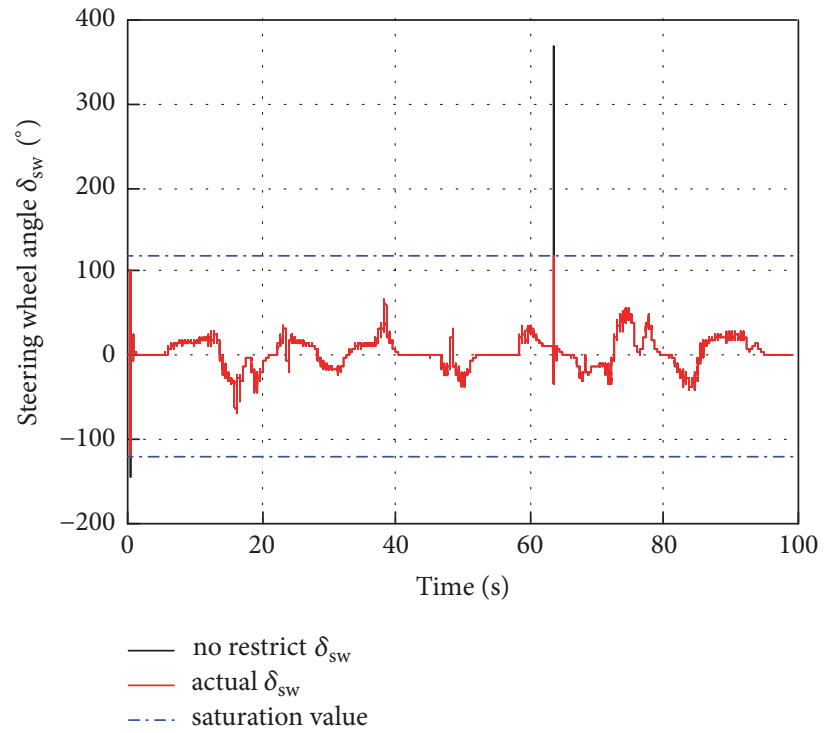

(d)

FIgURE 18: Simulation results. (a) Tracking. (b) Lateral acceleration. (c) Longitudinal speed. (d) Steering wheel corner.

\section{References}

[1] S. Li, X. Gang, and H. Yu, "Three - degree of freedom model and steering characteristic simulation of vehicle maneuvering Dynamics," Mechanical Design and Manufacturing, vol. 3, pp. 260-264, 2015.

[2] W. Zhang, Research on several problems of vehicle active safety technology under machine vision perception [master's thesis], Shan Dong University, Jinan, China, 2015.

[3] H. Han, Research on vehicle speed measurement of urban environment based on intelligent perception [master's thesis], Shanghai Traffic University, Shanghai, China, 2014.

[4] H.-Y. Cheng, C.-C. Yu, C.-C. Tseng, K.-C. Fan, J.-N. Hwang, and B.-S. Jeng, "Environment classification and hierarchical lane detection for structured and unstructured roads," IET Computer Vision, vol. 4, no. 1, pp. 37-49, 2010.
[5] Y. Dai, Vertical control of distributed electric drive vehicle [master's thesis], Tsinghua University, Beijing, China, 2013.

[6] S. Wei and X. Zhou, Multi-attribute decision-making theory and its application in C3I system [master's thesis], National Defense Industry Press, Beijing, China, 1998.

[7] C. Sentouh, P. Chevrel, F. Mars, and F. Claveau, "A sensorimotor driver model for steering control," in Proceedings of the 2009 IEEE International Conference on Systems, Man and Cybernetics, SMC 2009, pp. 2462-2467, IEEE Press, San Antonio, Tex, USA, October 2009.

[8] H.-H. Yang and H. Peng, "Development of an errorable carfollowing driver model," Vehicle System Dynamics, vol. 48, no. 6, pp. 751-773, 2010.

[9] A. Gray, Y. Gao, J. K. Hedrick, and F. Borrelli, "Robust predictive control for semi-autonomous vehicles with an uncertain driver 
model," in Proceedings of the IEEE Intelligent Vehicles Symposium (IV '13), pp. 208-213, Gold Coast, Australia, June 2013.

[10] C. I. Chatzikomis and K. N. Spentzas, "A path-following driver model with longitudinal and lateral control of vehicle's motion," Forschung im Ingenieurwesen, vol. 73, no. 4, pp. 257-266, 2009.

[11] L. Menhour, D. Lechner, and A. Charara, "Two degrees of freedom PID multi-controllers to design a mathematical driver model: Experimental validation and robustness tests," Vehicle System Dynamics, vol. 49, no. 4, pp. 595-624, 2011.

[12] H.-Z. Li, L. Li, J. Song, and L.-Y. Yu, "Comprehensive lateral driver model for critical maneuvering conditions," International Journal of Automotive Technology, vol. 12, no. 5, pp. 679-686, 2011.

[13] K. Mikami, H. Okuda, S. Taguchi, Y. Tazaki, and T. Suzuki, "Model predictive assisting control of vehicle following task based on driver model," in Proceedings of the 2010 IEEE International Conference on Control Applications, CCA 2010, pp. 890-895, Japan, September 2010.

[14] K. Guo, "Prediction of the large angle maneuvering motion of the closed loop system based on the theory of maneuvering," Automotive Engineering, vol. 01, pp. 1-11, 1992.

[15] K. Guo, F. Pan, Y. Cheng, and H. Ding, "Driver model based on the preview optimal artificial neural network," in Proceedings of the the 6th International Symposium on Advanced Vehicle Control (AVEC'02), 2002.

[16] F. Pan, Human-car closed-loop system driver neural network integrated optimization modeling [master's thesis], Jilin University, Changchun, China, 2001.

[17] Y. Cheng, The Driver model based on error analysis and its application in ADAMS [master's thesis], Jilin University, Changchun, China, 2003.

[18] F. Wan, Neural network driver model for vehicle handling stability evaluation [master's thesis], Jilin University, Changchun, China, 2005.

[19] H. Ding, K. Guo, F. Li, and J. Zhang, "Arbitrary path and speed following driver model based on vehicle acceleration feedback," Journal of Mechanical Engineering, vol. 46, no. 10, pp. 116-120, 2010.

[20] Y. Liu, Research on modulation strategy of dual-phase permanent magnet synchronous starting generator for electric vehicle [master's thesis], Harbin Institute of Technology, Harbin, China, 2014.

[21] L. Zhang, Research on unified decision model of vehicle driver's control behavior [master's thesis], Jilin University, Changchun, China, 2007.

[22] J. E. A. Dias, G. A. S. Pereira, and R. M. Palhares, "Longitudinal Model Identification and Velocity Control of an Autonomous Car," IEEE Transactions on Intelligent Transportation Systems, vol. 16, no. 2, pp. 776-786, 2015.

[23] Y. Li, Direction and speed integrated control of driver model and its application in ADAMS [master's thesis], Jilin University, Changchun, China, 2008.

[24] H. Ding, K. Guo, F. Li, and J. Zhang, "Arbitrary path and speed following driver model based on vehicle acceleration feedback," Journal of Mechanical Engineering, vol. 46, no. 10, pp. 116-125, 2010.

[25] M. Yamakado and M. Abe, "An experimentally confirmed driver longitudinal acceleration control model combined with vehicle lateral motion," Vehicle System Dynamics, vol. 46, no. 1, pp. 129149, 2008.

[26] Y. Koh, H. Her, K. Yi, and K. Kim, "Integrated speed and steering control driver model for vehicle-driver closed-loop simulation,"
IEEE Transactions on Vehicular Technology, vol. 65, no. 6, pp. 4401-4411, 2016.

[27] C. Han, Remote sensing control analysis and design [master's thesis], Xian Jiaotong University Press, Xi'an, China, 2011. 


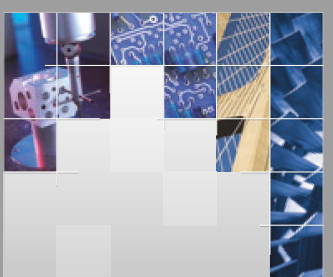

\section{Enfincering}
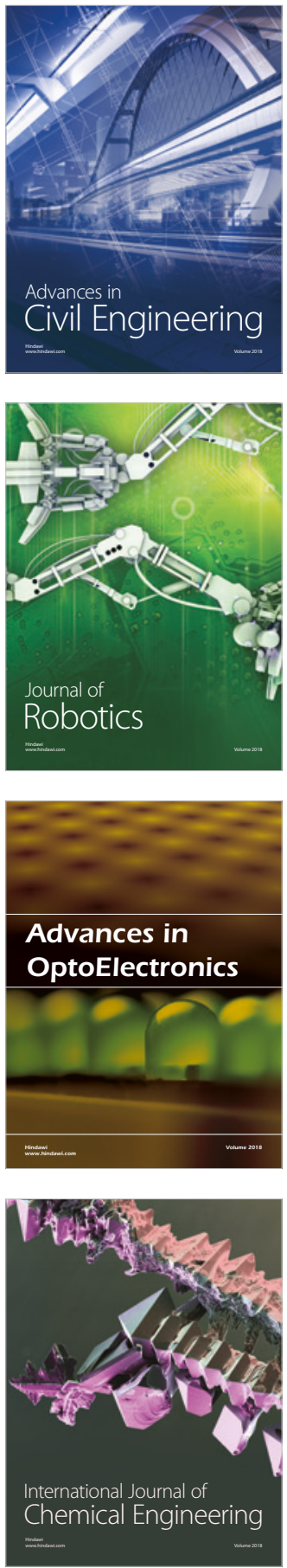

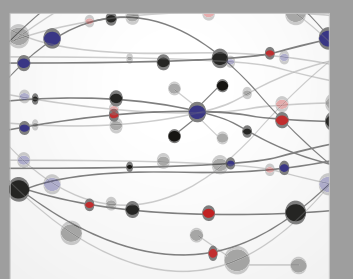

\section{Rotating \\ Machinery}

The Scientific World Journal

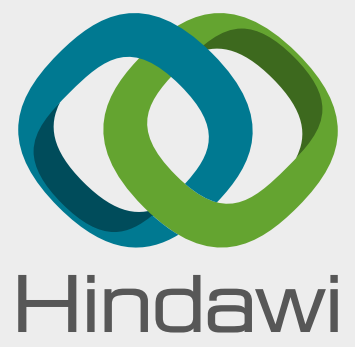

Submit your manuscripts at

www.hindawi.com
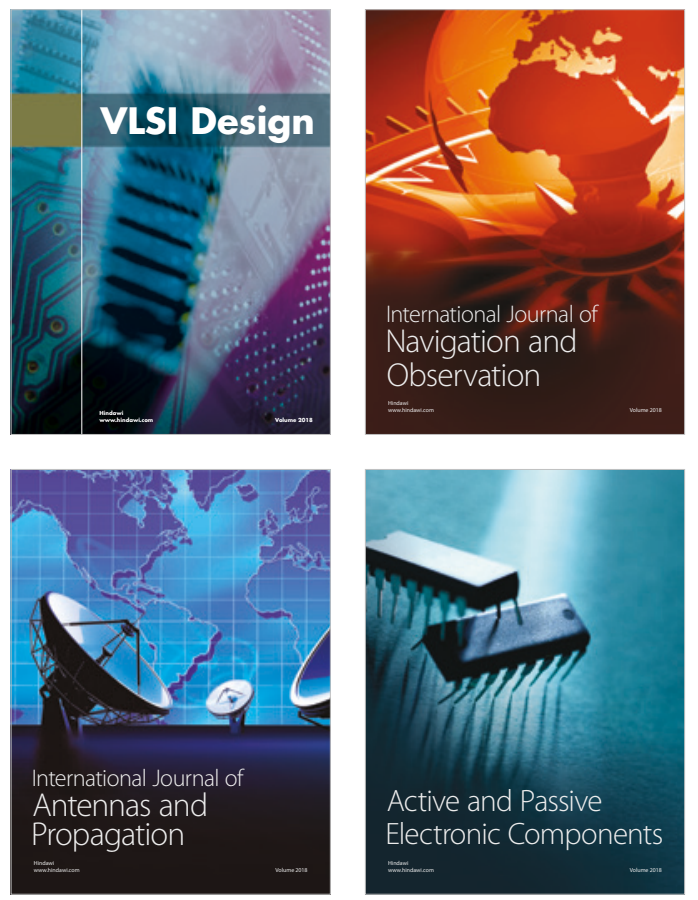
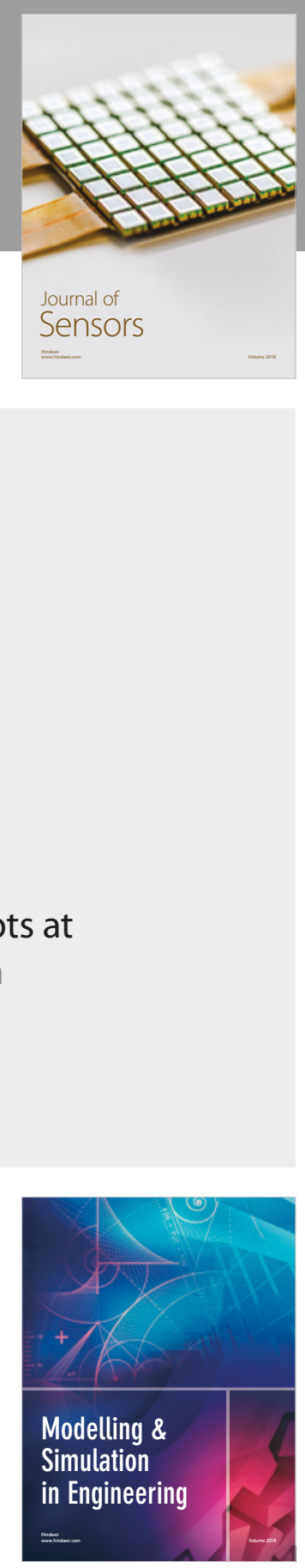

\section{Advances \\ Multimedia}
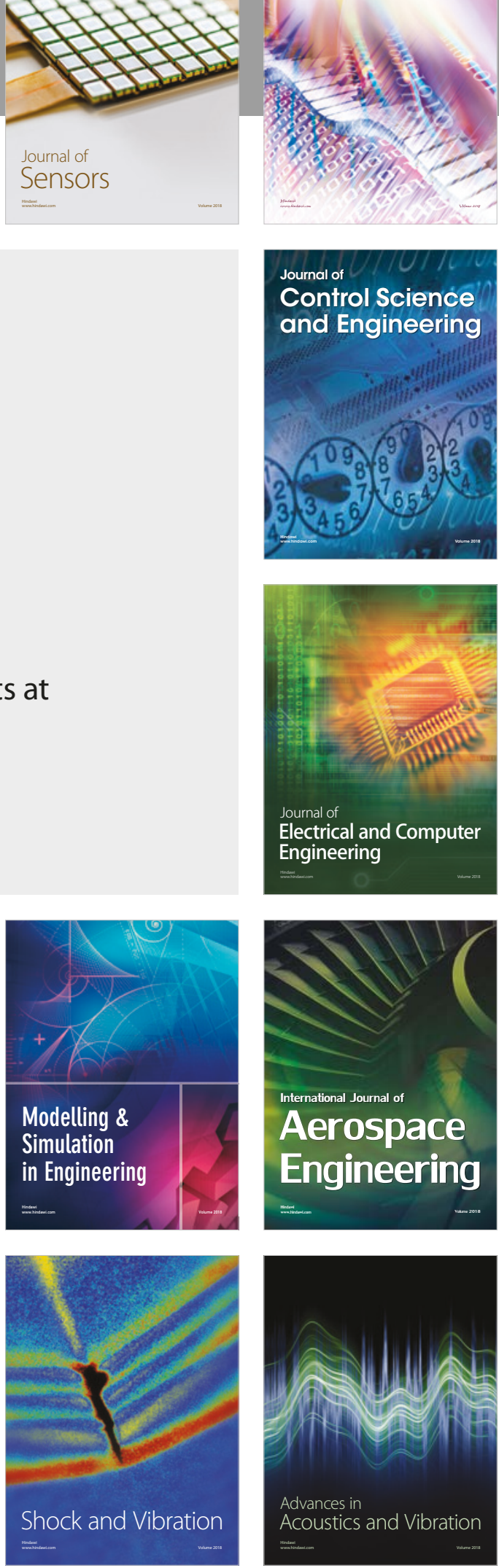